\title{
Three-Dimensional Echographic Evaluation of Carotid Artery Disease
}

\author{
Enrico Calogero ${ }^{1,2}$, Iacopo Fabiani ${ }^{1,2}$, Nicola Riccardo Pugliese ${ }^{1,2}$, Veronica Santini ${ }^{3}$, Lorenzo Ghiadoni ${ }^{3}$, Rossella Di Stefano ${ }^{1,2}$, Fabio Galetta ${ }^{3}$, \\ Ferdinando Sartucci ${ }^{3}$, Giuseppe Penno ${ }^{3}$, Raffaella Berchiolli ${ }^{4}$, Mauro Ferrari ${ }^{4}$, Dania Cioni ${ }^{4}$, Vinicio Napoli ${ }^{4}$, Raffaele De Caterina ${ }^{1,2}$, Vitantonio Di Bello ${ }^{1,2}$, \\ Davide Caramella ${ }^{4}$ \\ Departments of ${ }^{1}$ Medical, Surgical, Molecular and Critical Area Pathology, ${ }^{2}$ Cardiac, Thoracic and Vascular, ${ }^{3}$ Clinical and Experimental Medicine and ${ }^{4}$ Translational \\ Research and New Technologies in Medicine and Surgery, Pisa University, Pisa, Italy
}

\section{Abstract}

The introduction of three-dimensional echography (3D echo) in vascular field is not recent, but it still remains a seldom-used technique because of the costs of ultrasound probe and the need of dedicated laboratories. Therefore, despite significant prognostic implications, the high diagnostic accuracy in plaque definition, and the relative ease of use, $3 \mathrm{D}$ echo in vascular field is a niche technique. The purpose of this review is mainly clinical and intends to demonstrate the potential strength of a 3D approach, including technical aspects, in order to present to clinicians and imagers the appealing aspects of a noninvasive and radiation-free methodology with relevant diagnostic and prognostic correlates in the assessment of carotid atherosclerosis. A comprehensive literature search (since 1990s to date) using the PubMed, MEDLINE, and Cochrane libraries databases has been conducted. Articles written in English have been assessed, including reviews, clinical trials, meta-analyses, and interventional/observational studies. Manual cross-referencing was also performed, and relevant references from selected articles were reviewed. The search was limited to studies conducted in humans. Search terms, retrieved also with PubMed Advanced search and AND/OR Boolean operators (mainly in title and abstract), included three-dimensional, echo, stroke/transient ischemic attack, predictors, carotid, imaging, and biomarkers.

Keywords: Carotid atherosclerosis, color Doppler ultrasound, internal carotid artery stenosis, three-dimensional ultrasound imaging, total plaque volume

\section{INTRODUCTION}

Approximately $20 \%-30 \%$ of ischemic strokes are caused by the rupture of carotid artery plaques, and the percentage diameter reduction in the carotid arteries is used as an indicator for stroke risk. ${ }^{[1,2]}$ The risk factors leading to plaque rupture, however, remain incompletely understood. Stroke rates achieved after guideline-directed carotid endarterectomy (CEA) for high-grade carotid stenosis in symptomatic patients (stroke/transient ischemic attack [TIA]) are significantly lower than those observed with best medical therapy alone; at the same time, in asymptomatic patients, the majority of medically treated patients remain stroke free during follow-up. ${ }^{[3]}$ Moreover, in asymptomatic population, increasing degrees of stenosis have not been associated with a correspondingly increased risk for stroke/TIA. ${ }^{[4]}$ It is, therefore, important to identify additional markers to assess the risk of stroke and to identify patients who would benefit most from revascularization. ${ }^{[5]}$

\begin{tabular}{|l|l|}
\hline \multicolumn{2}{|c|}{ Access this article online } \\
\hline Quick Response Code: & Website: \\
\hline & www.jcecho.org \\
\cline { 2 - 2 } & \\
\hline
\end{tabular}

Magnetic resonance imaging (MRI) is a sensitive and specific method for determining the plaque characteristics. ${ }^{[6,7]}$ At present, computed tomography angiography is not much accurate in delineating plaque composition. It is, however, useful to identify the luminal surface outline. ${ }^{[8,9]}$ Duplex ultrasonography (DUS) is the most widely available and low-risk noninvasive test to assess carotid plaque severity, although its correlation with angiographically measured stenosis severity is low. ${ }^{[10]}$ Two-dimensional (2D) B-mode imaging can visualize a number of anatomic features of the arterial wall and plaque. An important strength of ultrasonography is its ability to provide real-time physiologic

Address for correspondence: Dr. Nicola Riccardo Pugliese, Department of Cardiac, Thoracic and Vascular, Azienda Ospedaliero-Universitaria Pisana, Via Paradisa, Pisa 256124, Italy. E-mail: n.r.pugliese88@gmail.com

This is an open access journal, and articles are distributed under the terms of the Creative Commons Attribution-NonCommercial-ShareAlike 4.0 License, which allows others to remix, tweak, and build upon the work non-commercially, as long as appropriate credit is given and the new creations are licensed under the identical terms.

For reprints contact: reprints@medknow.com

How to cite this article: Calogero E, Fabiani I, Pugliese NR, Santini V, Ghiadoni L, Di Stefano R, et al. Three-dimensional echographic evaluation of carotid artery disease. J Cardiovasc Echography 2018;28:218-27. 
information on blood flow (Doppler methods). While the image quality of ultrasound and the observable anatomic detail continues to improve, it is still limited by interobserver variability. The addition of three-dimensional (3D) imaging protocols has attempted to address that limitation. ${ }^{[11]}$

The noninvasive morphologic features of pre-embolic unstable plaques and their correlation with stroke still remain ill defined, due to a low incidence of stroke in asymptomatic patients with carotid plaques. Therefore, there continues to be a need to develop and validate plaque features that will identify patients at risk for future plaque disruption and athero-embolic cerebral infarction. In the past three decades, there has been a significant increase in the use of 3D carotid ultrasound for estimating the carotid plaque burden (CPB) through the estimation of luminal cross-sectional area.

The purpose of this review is mainly clinical and intends to demonstrate the potential strength of a 3D approach, including technical aspects, in order to present to clinicians and imagers the appealing aspects of a noninvasive and radiation-free methodology with relevant diagnostic and prognostic correlates in the assessment of carotid atherosclerosis.

\section{Searching Strategy}

A comprehensive literature search (since 1990s to date) using the PubMed, MEDLINE, and Cochrane libraries databases has been conducted. Articles written in English have been assessed, including reviews, clinical trials, meta-analyses, and interventional/observational studies. Manual cross-referencing was also performed, and relevant references from selected articles were reviewed. The search was limited to studies conducted in humans. Search terms, retrieved also with PubMed Advanced search and AND/OR Boolean operators (mainly in title/abstract), included three-dimensional, echo, stroke/TIA, predictors, carotid, imaging, and biomarkers.

\section{HISTORY}

From an historical perspective, in 1994, a volumetric method was introduced to assess plaque burden [Table 1]. ${ }^{[12]}$ Initial evaluations of carotid plaque volume were difficult; the disk segmentation method required collection of a set of 2D cross-sectional slices captured by moving the ultrasound probe on the neck along the carotid artery with a mechanical device. The plaque area was traced using continuous cross sections of the artery, stepping through the plaque at intervals of approximately $1 \mathrm{~mm}$, and the slices were summed to calculate the plaque volume. Early work specified the inter slice distance, reliability, reproducibility, and other technical details. ${ }^{[13-17]}$ Ludwig et al. ${ }^{[18]}$ in 2008 and following articles in $2014^{[19,20]}$ and $2015^{[21,22]}$ underlined good reproducibility and also discovered that reproducibility was superior for big plaques than for thin ones. The disk segmentation method was not only difficult, but also required 2-3 months of preparation. Initial semi-automated techniques ${ }^{[23,24]}$ reduced the number of slices of the plaque that needed manual input to establish the proximal and distal ends of each plaque. The operator had to delineate slices at the midpoint, $25 \%$ and $75 \%$ of the length of the plaque, with automated interpolation of the surface of the rest of the plaque. In 2013, an automatic technique was developed using mechanical movement of the probe along the artery ${ }^{[25]}$ that offered good agreement with manual segmentation by experts. However, the mechanism used to move the ultrasound probe is big and clumsy, and it is difficult to use in overweight patients and in patients with small necks. A mechanical sweep, acquired by keeping the probe in one site and having the angle changed mechanically, is quicker and more suitable. Recently, Græbe et al. ${ }^{[21]}$ showed that the mechanical sweep gave enhanced reproducibility of plaque volume determination compared with the manual movement of the probe along the artery.

\section{Three-Dimensional Ultrasonography: a Clinical Stand-Point}

From a clinical stand-point, the introduction of 3D imaging in the field of carotid ultrasounds stems at least from the following three main concepts:

1. The recognition of plaque pathophysiology, morphology, and its progression [Figures 1 and 2]

2. The importance of a direct evaluation of arteries (especially carotid) and tight follow-up besides the simple assessment of conventional risk factors, suggesting an "arterial target-based" instead of risk factor-based approach in cardiovascular (CV) preventive strategies

3. The inherent limitations in intimal media thickness (IMT) assessment and the potential implications for risk stratification reassessment (plaque burden).

Paired with the technological advances in 3D reconstruction and validation (i.e., magnetic resonance angiography [MRA]), together with an important reassessment of Doppler methodology, 3D carotid ultrasound is expected to contribute to the re-evaluation of the entire construct of the atherosclerotic process behind stroke/TIA and CV risk stratification.

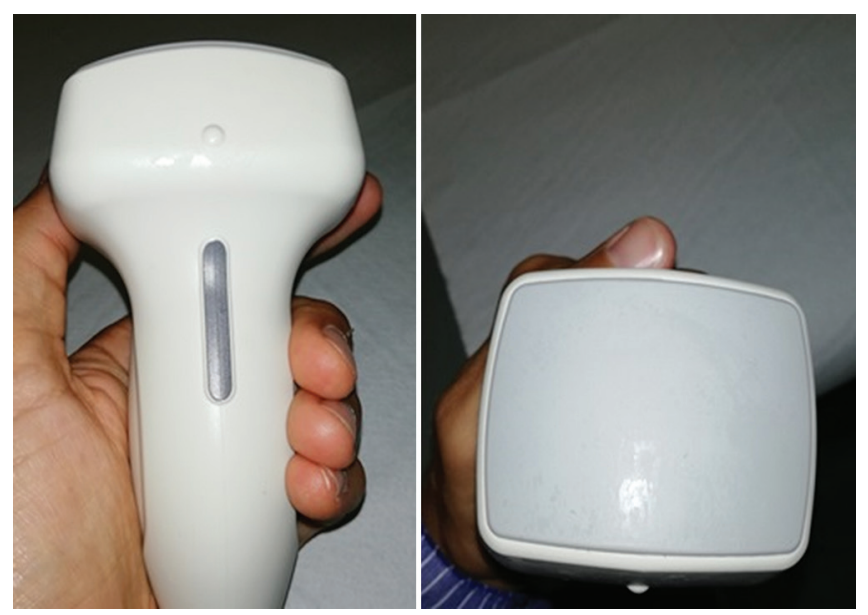

Figure 1: Three-dimensional vascular probe-automated mechanical sweep echographic probe 


\begin{tabular}{|c|c|c|c|c|}
\hline Year & Authors & Title & Study & Comments \\
\hline 1994 & Delcker A, Diener HC & $\begin{array}{l}\text { "Quantification of } \\
\text { atherosclerotic plaques } \\
\text { in carotid arteries by 3D } \\
\text { US" }\end{array}$ & $\begin{array}{l}\text { The aim of this study was to develop } \\
\text { 3D US imaging of the carotid arteries } \\
\text { in order to reduce intraexaminer and } \\
\text { interexaminer variabilities of volume } \\
\text { measurements in follow-up scans of } \\
\text { atherosclerotic plaques }\end{array}$ & $\begin{array}{l}\text { The newly developed 3D } \\
\text { computer-based image } \\
\text { reconstruction (disk segmentation) } \\
\text { allows repeated quantification of } \\
\text { moderate atherosclerosis with a } \\
\text { high reliability }\end{array}$ \\
\hline 2007 & $\begin{array}{l}\text { Landry A, } \\
\text { Ainsworth C, Blake C, } \\
\text { et al. }\end{array}$ & $\begin{array}{l}\text { "Manual planimetric } \\
\text { measurement of carotid } \\
\text { PV using 3D US } \\
\text { imaging" }\end{array}$ & $\begin{array}{l}\text { They investigated the utility of three } \\
\text { manual planimetric methods to } \\
\text { quantify carotid PV. Individual PVs } \\
\text { were using a standard planimetric } \\
\text { approach (M1), whereby a plaque } \\
\text { end was identified and sequential } \\
\text { contours were measured. The same }\end{array}$ & $\begin{array}{l}\text { M2 was determined to be a more } \\
\text { superior measurement technique } \\
\text { than M1 to measure individual PV. } \\
\text { Furthermore, they demonstrated the } \\
\text { utility of M3 to quantify regional } \\
\text { plaque burden and to quantify } \\
\text { change in PV }\end{array}$ \\
\hline
\end{tabular}

2011 Ukwatta E, Awad J, Ward $\mathrm{AD}$, et al.

\section{Buchanan D,} Gyacskov I, Ukwatta E, et al.

\author{
"3D US of carotid \\ atherosclerosis: \\ semiautomated \\ segmentation using a \\ level set-based method"
}

"Semi-automated segmentation of carotid artery TPV from 3D US carotid imaging" plaques were measured using a second approach (M2), whereby plaque ends were first identified and the 3D US image of the plaque was then subdivided into equal intervals. A third method (M3) was used to measure total plaque burden in a region relative to the carotid bifurcation

3D US of the carotid artery provides measurements of arterial wall and plaque (VWV) that are complementary to the one-dimensional measurement of the carotid artery intima-media thickness. 3D US VWV requires an observer to delineate the media-adventitia boundary and lumen-intima boundary of the carotid artery

Carotid artery TPV is a 3D US imaging measurement of carotid atherosclerosis, providing a direct noninvasive and regional estimation of atherosclerotic PV. While 3D US measurements of TPV provide the potential to monitor plaque in individual patients and in populations enrolled in clinical trials, until now, such measurements have been performed manually which is laborious, time consuming, and prone to intra- and inter-observer variability. To address this critical translational limitation, here, we describe the development and application of a semi-automated 3DUS PV measurement

Automatic segmentation of the carotid plaques from US images has been shown to be an important task for monitoring the progression and regression of carotid atherosclerosis. Considering the complex structure and heterogeneity of plaques, a fully automatic segmentation method based on media-adventitia and lumen-intima boundary priors is proposed. This method combines image intensity with structure information in both initialization and a level-set evolution process

\section{Comments}

computer-based image moderate atherosclerosis with a high reliability Furthermore, they demonstrated the plaque burden and to quantify

The proposed 3D semi-automated segmentation algorithm yielded high accuracy and high repeatability, while reducing the expert interaction required for initializing the algorithm than the previous $2 \mathrm{D}$ methods

They developed a semi-automated segmentation tool to measure $3 \mathrm{D}$ US TPV. The geometric volume approximation - this tool may provide a more accurate estimate compared to edge detection methods because signal void and artifacts, common in 3D US carotid images, would not influence contour shape and plaque morphology using a geometric method

Moreover, high correlation coefficients in generating TPV (0.993 and 0.992) between algorithm results and both sets of manual results were obtained. The automatic method provides a reliable way to segment carotid plaque in 3D US images and can be used in clinical practice to estimate plaque measurements for the management of carotid atherosclerosis

\footnotetext{
$3 \mathrm{D}=$ Three dimensional, $\mathrm{TPV}=$ Total plaque volume, $\mathrm{VWV}=$ Vessel wall volume, US=Ultrasound, DUS=Duplex ultrasonography, $\mathrm{PV}=\mathrm{Plaque}$ volume
} 


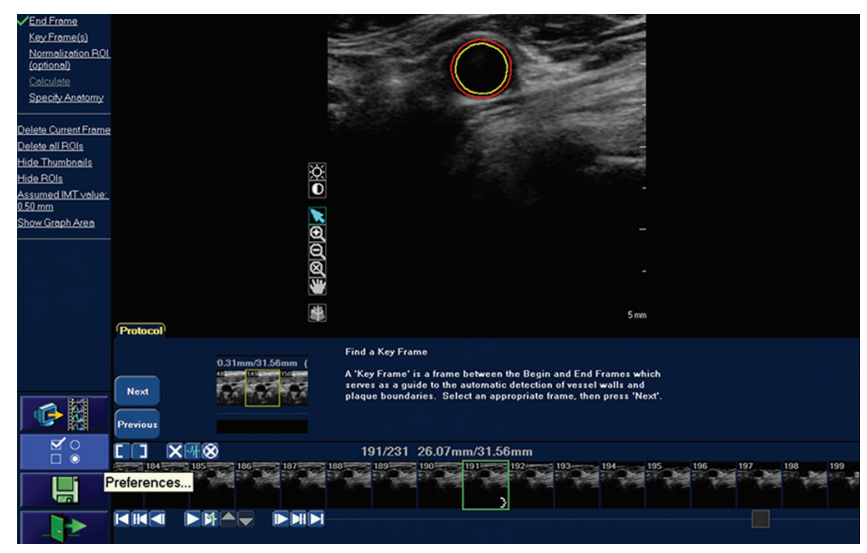

Figure 2: Vascular Plaque Quantification Software Offline (PACS) advanced analysis for total plaque volume and maximum percentage area reduction definition

\section{Total Plaque Volume and Prognosis/Risk Stratification}

Atherosclerotic plaque is a 3D phenomenon that is irregular in shape, and measurements performed solely in a $2 \mathrm{D}$ plane may miss the true extent of the plaque if it is out of plane. Compared with 2D imaging, 3D quantification of carotid plaque was shown to be more sensitive for clinically important angiographic stenosis in symptomatic patients. Plaques progress along the vessel is 2.4 times faster than the thickening of the wall. ${ }^{[26]}$ Therefore, methods that capture both longitudinal and circumferential growths (i.e., area and volume) are inherently more sensitive than methods limited to thickness measurements (i.e., diameter-reducing stenosis). The 5-year risk of stroke, myocardial infarction (MI), and vascular death is $19 \%$ for plaques with a longitudinal sectional area of $1.2-6.7 \mathrm{~cm}^{2}$ compared to $6 \%$ for areas of $0-0.1 \mathrm{~cm}^{2}$. Therefore, plaque area and volume (rather than diameter-reducing stenosis) may be better predictors of future rupture.

After adjusting for age, cholesterol, blood pressure, sex, homocysteine, smoking, diabetes, and treatment of cholesterol and blood pressure, patients in the top quartile of total plaque area (TPA) had a three times higher 5-year risk of death, stroke, or MI. ${ }^{[27,28]}$ In each quartile of TPA, the 5-year risks were approximately $5 \%, 10 \%, 15 \%$, and $20 \%$. Therefore, TPA was better than the Framingham risk profile in predicting risk. During the $1^{\text {st }}$ year of follow-up, almost half of the patients had plaque progression, a quarter had retrogression, and a quarter was constant. Those with plaque development had twice the risk of events, after adjustment for the group of risk factors listed before. TPA significantly improves risk prediction ${ }^{[28]}$ when added to risk calculation using scores based on risk factors [Table 2].

AlMuhanna et al. investigated the variability, reliability, and the least amount of change detectable by one-dimensional plaque measures, as well as 2D and 3D measures of plaque morphometry, that can be applied in a clinical environment in patients with carotid stenosis. ${ }^{[22]}$ They found carotid plaque diameter measurements from B-mode images as they have a high variability while plaque burden resulted readily and reliably measurable with a 3D US technique using a clinical scanner.

In 2011, Makris et al..$^{[1]}$ performed a systematic review of seven studies on the reproducibility of plaque volume reported an overall good intra- and inter-observer reproducibility. In addition, in ten further studies evaluating 3D plaque progression, volume performed better than IMT for plaque assessment in response to therapy [Table 3].

Importantly, the time taken to complete the quantification was not longer than 10-15 min. These developments increase the possibility for $3 \mathrm{D}$ quantification of carotid plaques to reach the clinical environment. It reliably detects plaque volume changes as low as $4 \%-6 \%$ with $95 \%$ confidence. ${ }^{[29]}$ Wannarong measured TPA, total plaque volume (TPV), and IMT with 3D ultrasound in 349 patients and followed the patients annually for 5 years. Using Cox regression analysis, survival free of any $\mathrm{CV}$ events (stroke, vascular death, TIA, or MI) was predicted by TPV progression or regression. TPA was a predictor of stroke, death, and TIA, but not of CV events. Progression or regression of IMT thickness did not predict any of these events. $^{[1]}$

Carotid 3D plaque volume is also more sensitive to the effects of therapy than coronary intravascular ultrasound (IVUS), whereas carotid plaques are focal and can change in three dimensions; coronary plaques are present along the entire length and entire circumference of the artery, so change is reduced to a change in average thickness.

A study on the effects of statins on plaque progression/regression indicates that change in TPA or TPV can be observed after 3 months. ${ }^{[30]}$

Sillesen et al. ${ }^{[26]}$ in 2012 studied 6101 asymptomatic patients without known CV disease with a 3D-based ultrasound approach (plaque burden), comparing CPB, carotid intima-media thickness (cIMT), ankle-brachial index (ABI), and abdominal aortic diameter (AAD) to coronary artery calcium score (CACS). In 3D, more than half of the patients had intermediate Framingham Risk Score (6\%-20\% 10-year risk). Carotid plaques were identified in $78 \%$ of cases and abnormal $\mathrm{ABI}$ in $10 \%$; $\mathrm{CPB}$ demonstrated a higher correlation with CACS than did CIMT, AAD, and ABI.

Johri et al. ${ }^{[31]}$ performed 2D and 3D carotid ultrasound scans on seventy consecutive patients referred for coronary angiography on the same day for plaque quantification in the carotid bulbs. $3 \mathrm{D}$ carotid ultrasound resulted in a higher negative predictive value and sensitivity relative to $2 \mathrm{D}$ carotid ultrasound and accurately predicted the absence of significant CAD.

Subsequently, in the BioImage Study (A Clinical Study of Burden of Atherosclerotic Disease in an At-Risk Population), the authors validated this approach in order to identify imaging biomarkers that predicted near-term (3-year) atherothrombotic events. ${ }^{[26]}$ Bilateral carotid artery plaque areas were summed 
Calogero, et al:: 3D carotid echo

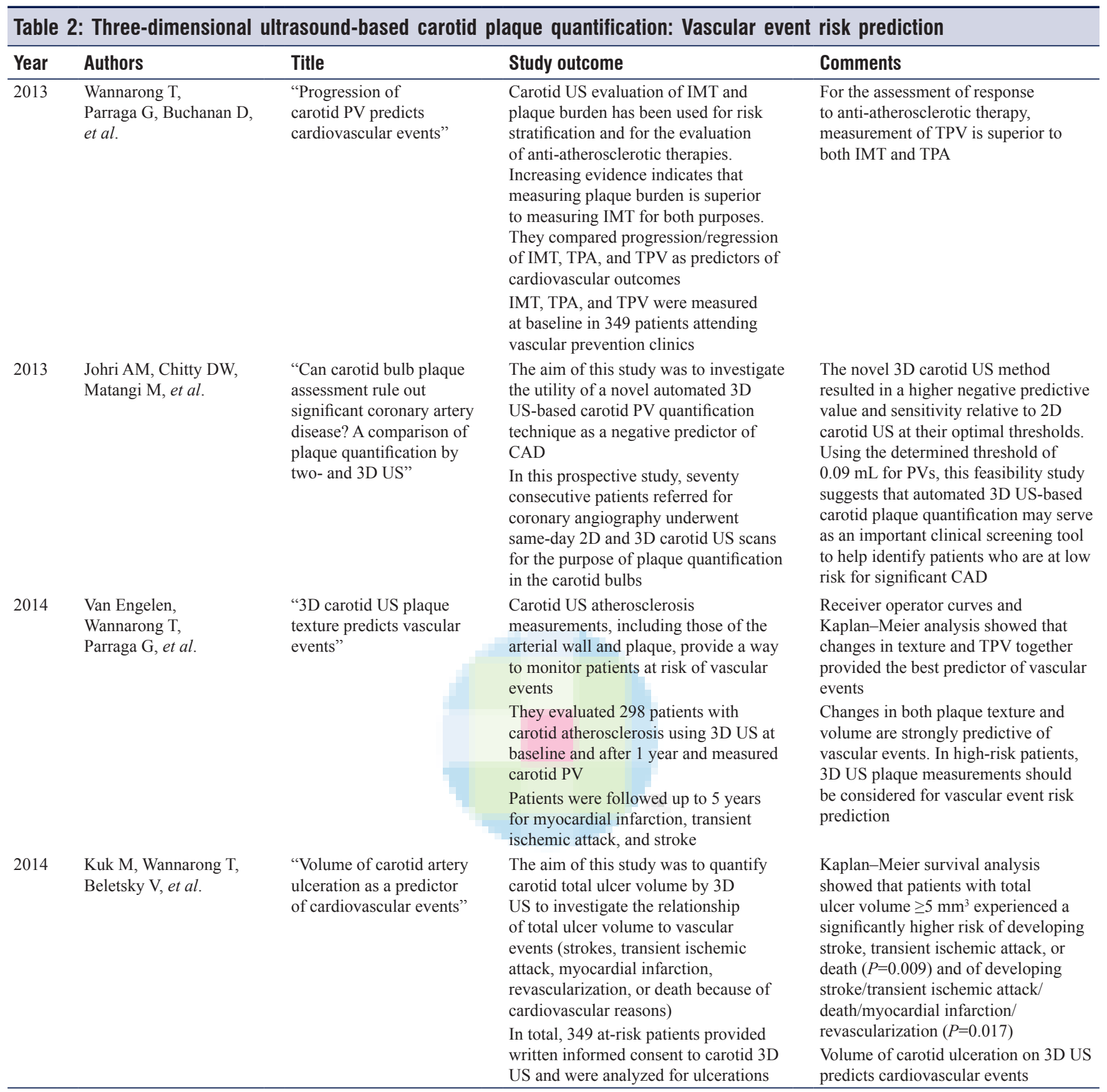

$3 \mathrm{D}=$ Three dimensional, $2 \mathrm{D}=$ Two dimensional, $\mathrm{CAD}=$ Coronary artery disease, IMT=Intima-media thickness, TPA=Total plaque area, $\mathrm{TPV}=\mathrm{Total}$ plaque volume, $\mathrm{PV}=\mathrm{Plaque}$ volume

to obtain a CPB. Over a median follow-up of 2.70 years, after correction for risk factors and matching with individuals without any $\mathrm{CPB}$, hazard ratios for MACE were 0.780 ( $95 \%$ confidence interval [CI]: 0.31-1.91), 1.45 (95\% CI: 0.670-3.140), and 2.36 (95\% CI: 1.13-4.92) in increasing CPB tertiles with comparable results for CACS. Net reclassification improvement appreciably improved with either CPB (0.23) or CACS $(0.25)$. MACE rates increased concurrently with higher levels of both CPB and CACS, thus showing that subclinical carotid or coronary atherosclerosis actually increases risk predictions and reclassification compared with traditional risk factors, with similar results for either modality.

\section{Vessel Wall Volume}

In patients without plaque, such as children and healthy volunteers, sometimes it has been a common practice to measure IMT. However, a viable alternative is the measurement of vessel wall volume (VWV, i.e., the volume of the artery minus the volume of the lumen). ${ }^{[32]}$ That measurement, which is conceptually equivalent to a 3D IMT, has significant advantages over IMT. First, like TPV, ${ }^{[33]}$ VWV has a much greater dynamic range; thus, it also potentially reduces sample size and duration of therapy compared with IMT and IVUS. Atorvastatin significantly reduced VWV in 3 months $^{[34]}$ in 
Calogero, et al:: 3D carotid echo

\begin{tabular}{|c|c|c|c|}
\hline Year & Authors & Title & Study \\
\hline 2004 & $\begin{array}{l}\text { Landry AM, Spence } \\
\text { JD, Fenster A. }\end{array}$ & $\begin{array}{l}\text { "Measurement of } \\
\text { carotid PV by 3D US" }\end{array}$ & $\begin{array}{l}\text { In this study, they investigated the } \\
\text { observer variability in the measurement } \\
\text { of PV as determined by 3D US. They } \\
\text { also investigated the effect of interslice } \\
\text { distances and repeated 3D US scans on } \\
\text { measurement variability }\end{array}$ \\
\hline
\end{tabular}

2008 Ludwig M, Zielinski T, Schremmer D, Stumpe $\mathrm{KO}$

2009 Mallett C, House AA, Spence JD, et al.

2014 Græbe M, Entrekin R, Collet-Billon A, Harrison G, Sillesen H.

"Reproducibility of two 3D US carotid plaque quantification methods"

"Reproducibility of 3 D US readings of volume of carotid atherosclerotic plaque"

The aim of this study was to investigate the quality of a central reading procedure concerning PV, measured by $3 \mathrm{D}$ US in a multinational US trial

"Longitudinal US Evaluation of Carotid Atherosclerosis in one, two and three dimensions"

2015 AlMuhanna K, Hossain MM, Zhao L, et al.

2015 Igase K, Kumon Y, Matsubara I, et al.
"Carotid plaque morphometric assessment with 3D US imaging"

"Utility of 3D US imaging to evaluate carotid artery stenosis: comparison with MRA"
The aim of this study was to compare the sensitivity of three US phenotypes of carotid atherosclerosis in a longitudinal study of patients with diabetic nephropathy. B-mode US-derived intima-media thickness, total plaque area, as well as 3D US VWV of the common carotid artery and internal carotid artery Compared with single 2D images, emerging 3D US technologies hold the promise of reducing the variability and increasing the sensitivity in the quantification of carotid plaques for individual cardiovascular risk stratification. Inter- and intra-observer agreement between a manual, cross-sectional, 2D freehand sweep and a mechanical 3D US investigation of 62 carotid artery plaques has been reported with intraclass correlation coefficients (with 95\% CIs)

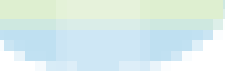

As investigations into nonsurgical treatment for atherosclerosis expand, the measurement of plaque regression and progression has become an important end are more reliable and sensitive to change than are traditional estimates of stenosis severity or cross-sectional area 3D US imaging may allow monitoring of PV changes but has not been used routinely due to the cumbersome motorized units required to drive transducers. They investigated the variability, reliability, and the least amount of change detectable by $1 \mathrm{D}$ plaque measures, as well as $2 \mathrm{D}$ and $3 \mathrm{D}$ measures of plaque morphometry that can be applied in a clinical environment They evaluated the utility of 3D US imaging for the assessment of carotid artery stenosis, as compared with similar assessment via MRA. Participants comprised 58 patients with carotid stenosis who underwent both 3D US imaging and MRA point to evaluate. Measurements of 3D PV

\section{Comments}

Intraobserver and interobserver measurement reliabilities were $94 \%$ and $93.2 \%$, respectively. PV measurement variability decreased with increasing PV (range, $27.1 \%-2.2 \%$ ). Repeated 3D US scan measurements were not different from single-scan measurements By implementing standardized central 3D US reading protocols and strict quality control procedures, highly reliable ultrasonic readings of plaque images can be achieved in large multicenter trials

These results suggest that $3 \mathrm{D}$ US-derived VWV provides necessary and sufficient sensitivity and specificity to measure longitudinal changes in small numbers of carotid atherosclerosis patients at risk of disease progression and over short periods of time

The use of semi-automated computerized planimetric measurements of plaque burden has high intraobserver repeatability, but is vulnerable to systematic interobserver differences. For the 2D freehand sweep, a considerable contribution to variation is introduced by the scanning procedure itself, that is, the lack of controlled motion along the third dimension. Future implementation of 3D US quantification in large-scale studies of inter-individual cardiovascular risk assessment seems justified using the methods described

Carotid plaque diameter measurements from B-mode images have high variability. Plaque burden, as estimated by VWV, can be measured reliably with a 3D US technique using a clinical scanner. The volumetric change, with $95 \%$ confidence, that must be observed to establish that a plaque has undergone growth or regression is $12.9 \%$ for different observers and $4.5 \%$ for the same observer performing the follow-up study

US imaging was necessary to detect mild stenosis, ulcerated plaque, or mobile plaque in comparison with MRA, and 3D US imaging was useful to recognize carotid stenosis and flow pattern as a total structure by static and real-time 3D demonstration

$3 \mathrm{D}=$ Three dimensional, 2D=Two dimensional, 1D=One dimensional, MRA=Magnetic resonance angiography, TPV=Total plaque volume, $\mathrm{VWV}=\mathrm{Vessel}$ wall volume, US=Ultrasound, $\mathrm{PV}=$ Plaque volume, $\mathrm{CIs}=$ Confidence intervals, DUS=Duplex ultrasonography 
the same patients in which TPV was measured in the study discussed previously. ${ }^{[35]}$ Dietary weight loss, probably through reduction of blood pressure, significantly reduced VWV in 2 years. ${ }^{[36]}$

\section{Ulceration, Echolucency, and Plaque Texture: approaching Plaque Composition}

An additional advantage of $3 \mathrm{D}$ imaging is an improved ability to evaluate the plaque surface. Ulceration, as detected by surface irregularity, is an important indicator of plaque instability, as it is strongly associated with the presence of rupture, plaque hemorrhage, large lipid core, less fibrous tissue, and overall instability. ${ }^{[37]}$ Angiographic studies of carotid arteries have shown that patients exhibiting ulceration and high stenosis $(85 \%)$ are twice as likely to have a stroke than patients who did not have ulcers; ${ }^{[38]}$ likewise, patients with carotid plaque surface irregularities are more likely to have a history of MI and to develop fatal acute MI, nonstroke vascular disorders, and sudden cardiac deaths than patients with smooth plaque. ${ }^{[39]}$ However, the accuracy of detecting ulceration by angiography is relatively low, with high rates of false positives and false negatives, ${ }^{[40]}$ and B-mode ultrasound has been found to be a more accurate method for ulcer detection. ${ }^{[41]}$

Kuk et al. ${ }^{[19]}$ quantified carotid global ulcer volume by $3 \mathrm{D}$ ultrasound to analyze the relationship of total ulcer volume to vascular events (MI, death because of CV reasons, strokes, TIA, revascularization, or death because of $\mathrm{CV}$ reasons). Ulcer volume was defined as a clear discontinuity in an atherosclerotic lesion, with a volume $\geq 1 \mathrm{~mm}^{3}$ as measured using traditional manual segmentation. The sum of the volumes of any ulcers seen in both carotids was the total ulcer volume. Participants were monitored for a 5-year follow-up. Participants with global ulcer volume $\geq 5 \mathrm{~mm}^{3}$ had a considerably greater risk of experiencing stroke, TIA, or death and non mi torna! the combined endpoint, including MI-related death and revascularization. Smaller ulcer volumes predict neither events nor ulcer depth.

Madani et al. recognized carotid ulcerations with 3D ultrasound in asymptomatic patients with carotid stenosis $>60 \%$. These authors also performed transcranial Doppler (TCD) to detect micro-embolization to the middle cerebral artery. Patients with $\geq 3$ ulcers were more likely to have a stroke or death during a 3 -year follow-up (18\% vs. $2 \%$ ). Furthermore, patients in whom TCD detected micro-emboli also had an elevated risk of stroke or death $(20 \%$ vs. $2 \%){ }^{[42]}$

$3 \mathrm{D}$ ultrasound is superior to $2 \mathrm{D}$ ultrasound in allowing the differentiation between ulceration and gaps between consecutive plaques adjacent to one another. ${ }^{[43]} 3 \mathrm{D}$ ultrasound has been used to detect changes in the morphology of ulcers by comparing the 3D surface features of ulcerated plaque: ${ }^{[4]}$ the identification and quantification of ulcers by $3 \mathrm{D}$ ultrasound in association with carotid stenosis $(60 \%)$ has demonstrated a correlation between the number of ulcers (three or more) and the risk of stroke or death. ${ }^{[45]}$ Computer-assisted methods for the analysis of ultrasound imaging are constantly evolving for achieving greater accuracy in plaque quantitative analysis. Chiu et al. ${ }^{[46]}$ demonstrated that mean and Gaussian curvatures can be used to identify ulcers from in vivo 3D ultrasound images; the algorithm was validated using synthetic surfaces and vascular phantoms containing "ulcers" that were 2-4 mm in diameter. In 124 patients, the total ulcer volume was found to be predictive of a higher risk for CVD events. ${ }^{[40]}$ Quantification of plaque volume and ulceration can be conducted with most commercially available software, but there is currently no standard procedure for measuring ulceration by either $2 \mathrm{D}$ or 3D ultrasound, and as suggested by Muraki et al., ${ }^{[47]}$ current classification criteria may be too conservative for accurate ulcer detection. It has long been thought that echolucent plaque represented "soft plaque" that was more likely to rupture and embolize. Several studies have shown that echolucency predicted higher risk of CV events. ${ }^{[48-50]}$ More recently, radiofrequency analysis (as opposed to gray-scale analysis) has been used to study plaque "texture," a way of assessing plaque composition. ${ }^{[51,52]}$ Inhomogeneity of plaque texture and some other features of plaque texture have been shown to identify patients at higher risk of events. ${ }^{[3,54]}$ Noninvasive characterization of intraplaque hemorrhage and enlarging lipid cores may identify individuals at risk for stroke. 2D longitudinal sectional B-mode images have been used to predict plaque histology, but a single slice provides limited information. Tissue characterization from 3D ultrasound may offer more comprehensive information. This approach may improve risk stratification, monitoring for the effectiveness of pharmacologic management, and selection of patients for carotid revascularization. ${ }^{[42]}$

Van Engelen et al. examined carotid ultrasound plaque texture and its changes in 298 patients at risk of events and compared them with the measurements of plaque volume and other risk factors as predictors of vascular events during 1 year. Patients were followed up to 5 years for MI, TIA, and stroke. In multivariate Cox regression analysis, changes in plaque texture and TPV were both significant predictors, whereas the Framingham risk score was not. ${ }^{[20]}$

\section{Performance of Three-Dimensional versus Other Methodologies (Angiography and Magnetic Resonance Imaging)}

Yao et al. studied 14 patients both with 3D ultrasound and carotid angiography (CA). A 3D ultrasound examination was performed also after surgery. The volume and length of twenty randomly selected lesions were measured from $3 \mathrm{D}$ data sets. The severity of stenosis was measured by $3 \mathrm{D}$ ultrasound using both the area and the diameter of several cross-sectional examinations at the most stenotic location; the results were then compared with CA, showing good correlation with $3 \mathrm{D}$ ultrasound in the quantitative analysis of carotid stenosis. ${ }^{[2]}$ 


\section{Calogero, et al.: 3D carotid echo}

In another article, ${ }^{[55]}$ the degree of luminal narrowing, measured as percentage area reduction in the $3 \mathrm{D}$ data set, correlated well with the degree of stenosis estimated by CA. In particular, 3D ultrasound showed good sensitivity and diagnostic accuracy for the detection of significant stenosis of extracranial carotid artery, showing, for stenosis between $40 \%$ and $70 \%$, a higher degree than CA.

In 2014, Pelz et al.$^{[56]}$ performed 3D ultrasound of carotid vessels including 3D ultrasound quantification of internal carotid artery (ICA) stenosis and contrast-enhanced-MRA(CE-MRA) of the neck vessels, showing good interrater and intermethod agreement in comparison to CE-MRA.

Chiu et al. ${ }^{[46]}$ developed an accurate surface-based 3D iterative closest point registration method to align surfaces reconstructed from outer wall boundaries segmented from 3D ultrasound and MRIs. The 3D ultrasound image was transformed according to the registration result and re-sliced to match the corresponding 2D transverse MRIs.

Vicenzini et al. ${ }^{[57]}$ described the use of this technique in conditions different than carotid stenosis, such as bifurcations and changes of the caliber or course of the neck vessels. In forty normal controls, seven patients with caliber alterations (four carotid bulb ectasia and three internal carotid lumen narrowings), 45 patients with course variations (tortuosity and kinking), and 35 patients with ICA stenosis of various degrees, they showed the feasibility and the improvement of carotid axis imaging through a better presentation of caliber variations and vessel course "at a glance."

Igase et al. ${ }^{[58]}$ evaluated the utility of $3 \mathrm{D}$ ultrasound for assessing carotid artery stenosis compared with MRA. In 58 patients with carotid stenosis, they showed how ultrasound imaging resulted useful in the detection of mild stenosis, ulcerated plaque, or mobile plaque in comparison with MRA.

\section{Three-Dimensional Doppler}

DUS has numerous pitfalls when the quantification of stenosis is attempted by spectral blood velocity parameters. ${ }^{[59-61]}$ In particular, for stenosis within the low $(<50 \%)$ to moderate $(50 \%-70 \%)$ range, intrastenotic velocity increases only slowly relative to the luminal loss, thereby resulting in a lack of diagnostic accuracy, that often requires additional morphological estimation of plaque stenosis in B-mode or color Doppler images, according to the North American Symptomatic Carotid Endarterectomy Trial method. ${ }^{[60-64]}$ Direct measurement of stenotic lumen reduction using 2D color Doppler ultrasound images delivered inconsistent results and may not improve accuracy when this method is used alone. ${ }^{[62,63]}$

Wessels et al. ${ }^{[65]}$ evaluated the intermethod agreement of ICA stenosis using digital subtraction angiography (DSA) compared with 3D ultrasound in 49 patients. Patients were investigated with a color Doppler system using the power mode. 3D ultrasound slightly underestimated the mean stenotic degree. The intermethod agreement comparing DSA with 3D echo was analyzed with the Bland and Altman test, which showed good agreement, yielding higher accuracy than CDS alone.

Stenosis grading with 3D echo using a dedicated volume probe generating static 3D images for immediate online and post hoc offline multiplanar rendering of relevant vessel segments, which is an innovative approach. The static 3D color Doppler images can be acquired after a quick topo-anatomic orientation with real-time four-dimensionally guided gray-scale imaging using the same probe. ${ }^{[59]}$

\section{Conclusions}

3D carotid ultrasound is an accurate and readily available method for atherosclerosis monitoring. It allows a fast and reproducible, volumetric, quantification of disease, consistent with a better risk stratification of individuals, without a significant slowdown of lab workflow. Validated against DSA and MRI, with the additional values of wall definition, plaque texture evaluation, and color Doppler all in one technique, it may provide a significant aid for redefinition of treatments in primary and secondary prevention, guiding different follow-up strategies.

\section{Acknowledgment}

We would like to thank Vassiliki Avramopoulou for her editorial assistance and English revision.

\section{Financial support and sponsorship}

The review is part of a project financed with Pisa University - PRA (Progetto di Ricerca di Ateneo) 2017 public fundings.

\section{Conflicts of interest}

There are no conflicts of interest.

\section{RefERENCES}

1. Makris GC, Lavida A, Griffin M, Geroulakos G, Nicolaides AN. Three-dimensional ultrasound imaging for the evaluation of carotid atherosclerosis. Atherosclerosis 2011;219:377-83.

2. Cires-Drouet RS, Mozafarian M, Ali A, Sikdar S, Lal BK. Imaging of high-risk carotid plaques: Ultrasound. Semin Vasc Surg 2017;30:44-53.

3. Demirel S, Böckler D, Storck M. Comparison of long-term results of carotid endarterectomy for asymptomatic carotid artery stenosis. Gefasschirurgie 2018;23:1-7.

4. Petty GW, Brown RD Jr., Whisnant JP, Sicks JD, O'Fallon WM, Wiebers DO, et al. Ischemic stroke subtypes: A population-based study of incidence and risk factors. Stroke 1999;30:2513-6.

5. Mughal MM, Khan MK, DeMarco JK, Majid A, Shamoun F, Abela GS, et al. Symptomatic and asymptomatic carotid artery plaque. Expert Rev Cardiovasc Ther 2011;9:1315-30.

6. Cai J, Hatsukami TS, Ferguson MS, Kerwin WS, Saam T, Chu B, et al. In vivo quantitative measurement of intact fibrous cap and lipid-rich necrotic core size in atherosclerotic carotid plaque: Comparison of high-resolution, contrast-enhanced magnetic resonance imaging and histology. Circulation 2005;112:3437-44.

7. Saam T, Ferguson MS, Yarnykh VL, Takaya N, Xu D, Polissar NL, et al. Quantitative evaluation of carotid plaque composition by in vivo MRI. Arterioscler Thromb Vasc Biol 2005;25:234-9.

8. Josephson SA, Bryant SO, Mak HK, Johnston SC, Dillon WP, Smith WS, et al. Evaluation of carotid stenosis using CT angiography in the initial evaluation of stroke and TIA. Neurology 2004;63:457-60.

9. Silvennoinen HM, Ikonen S, Soinne L, Railo M, Valanne L. CT 
angiographic analysis of carotid artery stenosis: Comparison of manual assessment, semiautomatic vessel analysis, and digital subtraction angiography. AJNR Am J Neuroradiol 2007;28:97-103.

10. Beach KW, Leotta DF, Zierler RE. Carotid Doppler velocity measurements and anatomic stenosis: Correlation is futile. Vasc Endovascular Surg 2012;46:466-74.

11. Naqvi TZ. Quantifying atherosclerosis by “3D” ultrasound works!: But there is work to be done. J Am Coll Cardiol 2015;65:1075-7.

12. Delcker A, Diener HC. Quantification of atherosclerotic plaques in carotid arteries by three-dimensional ultrasound. $\mathrm{Br} \mathrm{J}$ Radiol 1994;67:672-8.

13. Landry A, Fenster A. Theoretical and experimental quantification of carotid plaque volume measurements made by three-dimensional ultrasound using test phantoms. Med Phys 2002;29:2319-27.

14. Landry A, Spence JD, Fenster A. Measurement of carotid plaque volume by 3-dimensional ultrasound. Stroke 2004;35:864-9.

15. Landry A, Spence JD, Fenster A. Quantification of carotid plaque volume measurements using 3D ultrasound imaging. Ultrasound Med Biol 2005;31:751-62.

16. Landry A, Ainsworth C, Blake C, Spence JD, Fenster A. Manual planimetric measurement of carotid plaque volume using three-dimensional ultrasound imaging. Med Phys 2007;34:1496-505.

17. Mallett C, House AA, Spence JD, Fenster A, Parraga G. Longitudinal ultrasound evaluation of carotid atherosclerosis in one, two and three dimensions. Ultrasound Med Biol 2009;35:367-75.

18. Ludwig M, Zielinski T, Schremmer D, Stumpe KO. Reproducibility of 3-dimensional ultrasound readings of volume of carotid atherosclerotic plaque. Cardiovasc Ultrasound 2008;6:42.

19. Kuk M, Wannarong T, Beletsky V, Parraga G, Fenster A, Spence JD, et al. Volume of carotid artery ulceration as a predictor of cardiovascular events. Stroke 2014;45:1437-41.

20. van Engelen A, Wannarong T, Parraga G, Niessen WJ, Fenster A, Spence JD, et al. Three-dimensional carotid ultrasound plaque texture predicts vascular events. Stroke 2014;45:2695-701.

21. Græbe M, Entrekin R, Collet-Billon A, Harrison G, Sillesen H. Reproducibility of two 3-D ultrasound carotid plaque quantification methods. Ultrasound Med Biol 2014;40:1641-9.

22. AlMuhanna K, Hossain MM, Zhao L, Fischell J, Kowalewski G, Dux M, et al. Carotid plaque morphometric assessment with three-dimensional ultrasound imaging. J Vasc Surg 2015;61:690-7.

23. Ukwatta E, Awad J, Ward AD, Buchanan D, Samarabandu J, Parraga G, et al. Three-dimensional ultrasound of carotid atherosclerosis: Semiautomated segmentation using a level set-based method. Med Phys 2011;38:2479-93

24. Buchanan D, Gyacskov I, Ukwatta E, Lindenmaier T, Fenster A, Parraga G. Semi-automated segmentation of carotid artery total plaque volume from three dimensional ultrasound carotid imaging. Int Soc Opt Photonics 2012:83170I. Available from: http://proceedings. spiedigitallibrary.org/proceeding.aspx?doi $=10.1117 / 12.912880 . \quad$ [Last accessed on 2018 Jul 10].

25. Cheng J, Li H, Xiao F, Fenster A, Zhang X, He X, et al. Fully automatic plaque segmentation in 3-D carotid ultrasound images. Ultrasound Med Biol 2013;39:2431-46.

26. Sillesen H, Muntendam P, Adourian A, Entrekin R, Garcia M, Falk E, et al. Carotid plaque burden as a measure of subclinical atherosclerosis: Comparison with other tests for subclinical arterial disease in the high risk plaque BioImage study. JACC Cardiovasc Imaging 2012;5:681-9.

27. Johnsen SH, Mathiesen EB, Joakimsen O, Stensland E, Wilsgaard T, Løchen ML, et al. Carotid atherosclerosis is a stronger predictor of myocardial infarction in women than in men: A 6-year follow-up study of 6226 persons: The Tromsø study. Stroke 2007;38:2873-80.

28. Mathiesen EB, Johnsen SH, Wilsgaard T, Bønaa KH, Løchen ML, Njølstad I, et al. Carotid plaque area and intima-media thickness in prediction of first-ever ischemic stroke: A 10-year follow-up of 6584 men and women: The Tromsø study. Stroke 2011;42:972-8.

29. Hossain MM, AlMuhanna K, Zhao L, Lal BK, Sikdar S. Semiautomatic segmentation of atherosclerotic carotid artery wall volume using 3D ultrasound imaging. Med Phys 2015;42:2029-43.

30. Spence JD. Time course of atherosclerosis regression. Atherosclerosis 2014;235:347-8
31. Johri AM, Chitty DW, Matangi M, Malik P, Mousavi P, Day A, et al. Can carotid bulb plaque assessment rule out significant coronary artery disease? A comparison of plaque quantification by two- and three-dimensional ultrasound. J Am Soc Echocardiogr 2013;26:86-95.

32. Egger M, Spence JD, Fenster A, Parraga G. Validation of 3D ultrasound vessel wall volume: An imaging phenotype of carotid atherosclerosis. Ultrasound Med Biol 2007;33:905-14.

33. Pollex RL, Spence JD, House AA, Fenster A, Hanley AJ, Zinman B, et al. A comparison of ultrasound measurements to assess carotid atherosclerosis development in subjects with and without type 2 diabetes. Cardiovasc Ultrasound 2005;3:15.

34. Krasinski A, Chiu B, Spence JD, Fenster A, Parraga G. Three-dimensional ultrasound quantification of intensive statin treatment of carotid atherosclerosis. Ultrasound Med Biol 2009;35:1763-72.

35. Chimowitz MI, Weiss DG, Cohen SL, Starling MR, Hobson RW $2^{\text {nd }}$. Cardiac prognosis of patients with carotid stenosis and no history of coronary artery disease. Veterans affairs cooperative study group 167. Stroke 1994;25:759-65.

36. Shai I, Spence JD, Schwarzfuchs D, Henkin Y, Parraga G, Rudich A, et al. Dietary intervention to reverse carotid atherosclerosis. Circulation 2010;121:1200-8.

37. Levin DC, Fallon JT. Significance of the angiographic morphology of localized coronary stenoses: Histopathologic correlations. Circulation 1982;66:316-20.

38. Eliasziw M, Streifler JY, Fox AJ, Hachinski VC, Ferguson GG, Barnett HJ, et al. Significance of plaque ulceration in symptomatic patients with high-grade carotid stenosis. North American symptomatic carotid endarterectomy trial. Stroke 1994;25:304-8.

39. Rothwell PM, Villagra R, Gibson R, Donders RC, Warlow CP. Evidence of a chronic systemic cause of instability of atherosclerotic plaques. Lancet 2000;355:19-24.

40. Streifler JY, Eliasziw M, Fox AJ, Benavente OR, Hachinski VC, Ferguson GG, et al. Angiographic detection of carotid plaque ulceration. Comparison with surgical observations in a multicenter study. North American symptomatic carotid endarterectomy trial. Stroke 1994;25:1130-2.

41. Kagawa R, Moritake K, Shima T, Okada Y. Carotid surgery versus medical therapy in asymptomatic carotid stenosis. The CASANOVA study group. Stroke 1991;22:1229-35. Available from: http://www.ncbi. nlm.nih.gov/pubmed/1926232. [Last accessed on $2018 \mathrm{Jul} 12$ ].

42. Madani A, Beletsky V, Tamayo A, Munoz C, Spence JD. High-risk asymptomatic carotid stenosis: Ulceration on 3D ultrasound vs. TCD microemboli. Neurology 2011;77:744-50.

43. Heliopoulos J, Vadikolias K, Piperidou C, Mitsias P. Detection of carotid artery plaque ulceration using 3-dimensional ultrasound. J Neuroimaging 2011;21:126-31.

44. Yao J, van Sambeek MR, Dall'Agata A, van Dijk LC, Kozakova M, Koudstaal PJ, et al. Three-dimensional ultrasound study of carotid arteries before and after endarterectomy; analysis of stenotic lesions and surgical impact on the vessel. Stroke 1998;29:2026-31.

45. Barnett HJ, Eliasziw M, Meldrum HE, Taylor DW. Do the facts and figures warrant a 10-fold increase in the performance of carotid endarterectomy on asymptomatic patients? Neurology 1996;46:603-8.

46. Chiu B, Beletsky V, Spence JD, Parraga G, Fenster A. Analysis of carotid lumen surface morphology using three-dimensional ultrasound imaging. Phys Med Biol 2009;54:1149-67.

47. Muraki M, Mikami T, Yoshimoto T, Fujimoto S, Tokuda K, Kaneko S, et al. New criteria for the sonographic diagnosis of a plaque ulcer in the extracranial carotid artery. AJR Am J Roentgenol 2012;198:1161-6.

48. Polak JF, Shemanski L, O'Leary DH, Lefkowitz D, Price TR, Savage PJ, et al. Hypoechoic plaque at US of the carotid artery: An independent risk factor for incident stroke in adults aged 65 years or older. Cardiovascular health study. Radiology 1998;208:649-54.

49. Mazzone A, De Servi S, Ricevuti G, Mazzucchelli I, Fossati G, Pasotti D, et al. Increased expression of neutrophil and monocyte adhesion molecules in unstable coronary artery disease. Circulation 1993;88:358-63.

50. Hatsukami TS, Ross R, Polissar NL, Yuan C. Visualization of fibrous cap thickness and rupture in human atherosclerotic carotid plaque in vivo with high-resolution magnetic resonance imaging. Circulation 2000;102:959-64 
51. Kakkos SK, Nicolaides AN, Kyriacou E, Daskalopoulou SS, Sabetai MM, Pattichis CS, et al. Computerized texture analysis of carotid plaque ultrasonic images can identify unstable plaques associated with ipsilateral neurological symptoms. Angiology 2011;62:317-28.

52. Christodoulou CI, Pattichis CS, Pantziaris M, Nicolaides A. Texture-based classification of atherosclerotic carotid plaques. IEEE Trans Med Imaging 2003;22:902-12.

53. Kakkos SK, Stevens JM, Nicolaides AN, Kyriacou E, Pattichis CS, Geroulakos G, et al. Texture analysis of ultrasonic images of symptomatic carotid plaques can identify those plaques associated with ipsilateral embolic brain infarction. Eur J Vasc Endovasc Surg 2007;33:422-9.

54. Liapis CD, Kakisis JD, Kostakis AG. Carotid stenosis: Factors affecting symptomatology. Stroke 2001;32:2782-6.

55. Kozàkovà M, Morizzo C, Andreucetti F, Palchetti P, Parenti G, Palombo C, et al. Quantification of extracranial carotid artery stenosis by ultrafast three-dimensional ultrasound. J Am Soc Echocardiogr 2001;14:1203-11.

56. Pelz JO, Weinreich A, Fritzsch D, Saur D. Quantification of internal carotid artery stenosis with 3D ultrasound angiography. Ultraschall Med 2015;36:487-93.

57. Vicenzini E, Galloni L, Ricciardi MC, Pro S, Sirimarco G, Pulitano P, et al. Advantages and pitfalls of three-dimensional ultrasound imaging of carotid bifurcation. Eur Neurol 2011;65:309-16.

58. Igase K, Kumon Y, Matsubara I, Arai M, Goishi J, Watanabe H, et al. Utility of 3-dimensional ultrasound imaging to evaluate carotid artery stenosis: Comparison with magnetic resonance angiography. J Stroke Cerebrovasc Dis 2015;24:148-53.

59. Brott TG, Halperin JL, Abbara S, Bacharach JM, Barr JD, Bush RL, et al. 2011 ASA/ACCF/AHA/AANN/AANS/ACR/ASNR/CNS/ SAIP/SCAI/SIR/SNIS/SVM/SVS guideline on the management of patients with extracranial carotid and vertebral artery disease: A report of the American College of Cardiology Foundation/American Heart Association Task Force on Practice Guidelines, and the American Stroke Association, American Association of Neuroscience Nurses, American Association of Neurological Surgeons, American College of Radiology, American Society of Neuroradiology, Congress of Neurolog. Am Coll Cardiol 2011;57:e16-94. Available from: https://www.sciencedirect. com/science/article/pii/S0735109710045717. [Last accessed on 2018 Jul 14].

60. von Reutern GM, Goertler MW, Bornstein NM, Del Sette M, Evans DH, Hetzel A, et al. Grading carotid stenosis using ultrasonic methods. Stroke 2012;43:916-21.

61. Grant EG, Benson CB, Moneta GL, Alexandrov AV, Baker JD, Bluth EI, et al. Carotid artery stenosis: Gray-scale and Doppler US diagnosis - Society of radiologists in ultrasound consensus conference. Radiology 2003;229:340-6.

62. Beebe HG, Salles-Cunha SX, Scissons RP, Dosick SM, Whalen RC, Gale SS, et al. Carotid arterial ultrasound scan imaging: A direct approach to stenosis measurement. J Vasc Surg 1999;29:838-44.

63. Wardlaw JM, Lewis S. Carotid stenosis measurement on colour Doppler ultrasound: Agreement of ECST, NASCET and CCA methods applied to ultrasound with intra-arterial angiographic stenosis measurement. Eur J Radiol 2005;56:205-11.

64. Fox AJ. How to measure carotid stenosis. Radiology 1993;186:316-8.

65. Wessels T, Harrer JU, Stetter S, Mull M, Klötzsch C. Three-dimensional assessment of extracranial Doppler sonography in carotid artery stenosis compared with digital subtraction angiography. Stroke 2004;35:1847-51.

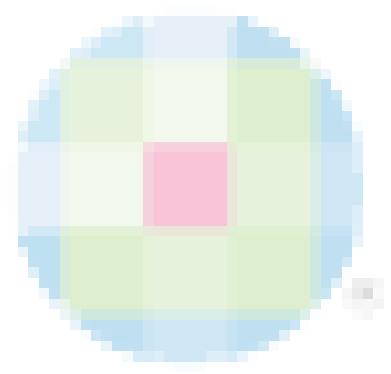

